\title{
Retroperitoneal abscess shortly after chemotherapy for lung cancer: A case report
}

\author{
GEN OHARA $^{1}$, TADASHI KONDO ${ }^{2}$, KATSUNORI KAGOHASHI ${ }^{1}$, HIROKO WATANABE $^{3}$, \\ MIO KAWAGUCHI ${ }^{3}$, KOICHI KURISHIMA ${ }^{1}$, HIROAKI SATOH ${ }^{1}$ and NOBUYUKI HIZAWA ${ }^{3}$
}

\author{
Divisions of ${ }^{1}$ Respiratory Medicine and ${ }^{2}$ Surgery, Mito Medical Center, University of Tsukuba, Mito, Ibaraki 310-0015; \\ ${ }^{3}$ Division of Respiratory Medicine, Faculty of Medicine, University of Tsukuba, Tsukuba, Ibaraki 305-8575, Japan
}

Received October 12, 2013; Accepted December 9, 2013

DOI: $10.3892 / \mathrm{mco} .2013 .228$

\begin{abstract}
To the best of our knowledge, the formation of a retroperitoneal abscess due to acute appendicitis shortly after administration of chemotherapy for lung cancer has not been previously reported. This is the case report of a 59-year-old male who was admitted to the Mito Medical Center (Mito, Japan) and diagnosed with lung adenocarcinoma with pleuritis carcinomatosis. Although no distant metastasis was identified, combination chemotherapy with cisplatin and pemetrexed was administered. Nine days after initiating chemotherapy, the patient developed right lower quadrant abdominal pain and high fever. Computed tomography (CT) of the abdomen and pelvis revealed the collection of gas and fluid in the retroperitoneum adjacent to the cecum. The abscess was locally drained; however, the infection continued to spread, with subsequent development of a scrotal abscess. Consequently, appendectomy was performed. The patient recovered well and the lung adenocarcinoma was treated with additional courses of chemotherapy following the remission of the local inflammation. Retroperitoneal abscess due to acute appendicitis is an unusual finding; however, this rare complication should be considered during or shortly after chemotherapy in patients with lung cancer.
\end{abstract}

\section{Introduction}

Retroperitoneal abscess is a rare but life-threatening infection, which may be caused by perforated or penetrated diverticulitis, ruptured appendix and inflammatory bowel disease (1-4). Appropriate medical and surgical management is required in order to reduce the associated high mortality rate. This is a rare case of retroperitoneal abscess, which continued to spread,

Correspondence to: Professor Hiroaki Satoh, Division of Respiratory Medicine, Mito Medical Center, University of Tsukuba, Miya-machi 3-2-7, Mito, Ibaraki 310-0015, Japan

E-mail: hirosato@md.tsukuba.ac.jp; kkagohashi1795@yahoo.co.jp

Key words: retroperitoneal abscess, chemotherapy, lung cancer eventually leading to the formation of a scrotal abscess, shortly after administration of chemotherapy for lung cancer.

\section{Case report}

A 59-year-old male, with no previous reported health issues, was referred to the Mito Medical Center (Mito, Japan) due to a persistent productive cough over the last few months. Chest radiography revealed a massive pleural fluid collection with a mediastinal shift (Fig. 1). On the basis of the results of a computed tomography (CT) scan and bronchoscopy, the patient was diagnosed with lung adenocarcinoma with pleuritis carcinomatosis, without any detected distant metastasis. Subsequently, combination chemotherapy with cisplatin and pemetrexed was administered. Nine days after the initiation of chemotherapy, the patient developed right lower quadrant abdominal pain and high fever. The blood examination data did not indicate leukopenia (white blood cell count, 7,500 cells $/ \mu \mathrm{l}$ ), but revealed a high $\mathrm{C}$-reactive protein (CRP) level $(16.7 \mathrm{mg} / \mathrm{dl})$. A CT scan revealed fluid and gas collection in the retroperitoneum adjacent to the cecum (Fig. 2). The appendix was not identified by imaging and, due to the presence of a cecal diverticulum, the patient was diagnosed with pericolic abscess and emergent drainage was performed. Administration of the meropenem and clindamycin was initiated, a catheter was inserted into the abscess and pus with gas and odor was drained, strongly suggesting an infection by gasproducing anaerobic microorganisms. The patient recovered temporarily; however, the infection continued to spread and a scrotal abscess developed (Fig. 3), although the size of the initial abscess had been reduced as a result of the drainage. X-ray imaging using iodinated contrast media revealed a fistular tract between the cecum and the abscess cavity. Perforated appendicitis was suspected and an appendectomy as performed. A microbiological examination of the pus revealed the presence of Escherichia coli, in addition to other anaerobic bacteria. The patient recovered well postoperatively and proceeded to receive treatment for the lung adenocarcinoma, achieving a partial response with additional courses of chemotherapy one month after the surgery. The operative findings indicated perforated appendicitis. Six months after the surgical treatment, the patient remains alive and is treated with chemotherapy in the outpatient clinic. 


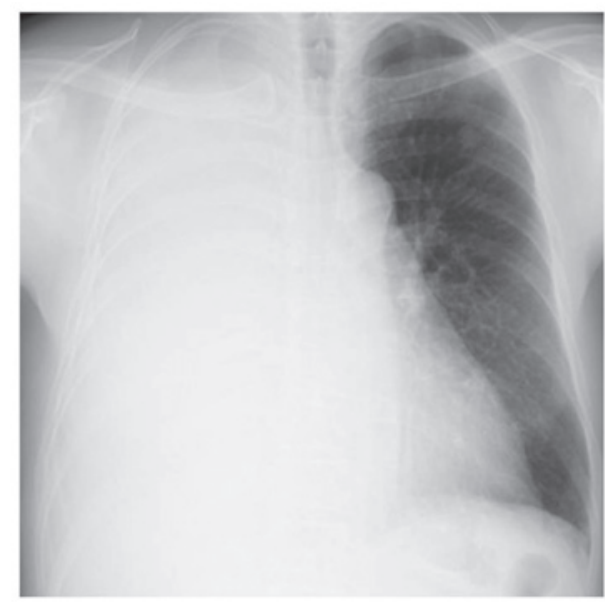

Figure 1. Chest radiography revealed a massive pleural fluid collection in the right hemithorax, with mediastinal shift.

\section{Discussion}

There have been several reports on the incidence of bowel perforation in patients with acute appendicitis or colon cancer resulting in the development of retroperitoneal, psoas, or scrotal abscesses $(1,5,6)$. Takakura et al $(6)$ reported a case of retroperitoneal abscess complicated by necrotizing fasciitis of the thigh in a patient with sigmoid colon cancer. The authors of that study reported that the abscess developed 4 months following the initiation of chemotherapy (6). Maguire and Kaye (7) reported two cases in which patients developed a psoas abscess after undergoing chemotherapy for bulky retroperitoneal deposits of teratoma. In those cases, the abscess developed 4 weeks after the last course of chemotherapy and 2 years after undergoing surgery and radiotherapy (7). In both cases, the primary lesion was located in the abdomen or in the retroperitoneal space. There was no chemotherapy-induced myelosuppression in those patients and their abscesses developed long after treatment (7). We also previously reported a case of retrocecal appendicitis, which resulted in perforation shortly after chemotherapy for lung cancer (8). In that case, the complication developed under conditions of leukocytopenia (white blood cell count, 500/ $/ \mathrm{l}$ ), but did not result in the formation of a retroperitoneal abscess (8). We successfully treated that condition with effective antibiotics and appendectomy after the recovery of leukocytopenia. We could not identify any case with retroperitoneal abscess during or shortly after chemotherapy for tumors originated in sites other than the abdomen or retroperitoneum. Therefore, to the best of our knowledge, this is the first case exhibiting development of retroperitoneal and scrotal abscesses due to the spread of the infection cause by perforated appendicitis, during or shortly after the administration of chemotherapy for lung cancer. The causes underlying the development of a complication such as retroperitoneal abscess shortly after chemotherapy for lung cancer have not been elucidated.

The clinical characteristics of retroperitoneal abscesses include high fever with chills and tenderness over the affected area, along with changes in skin color and palpable crepitus $(1,5,6)$. However, the insidious occult characteristics of these abscesses may sometimes lead to diagnostic delay.
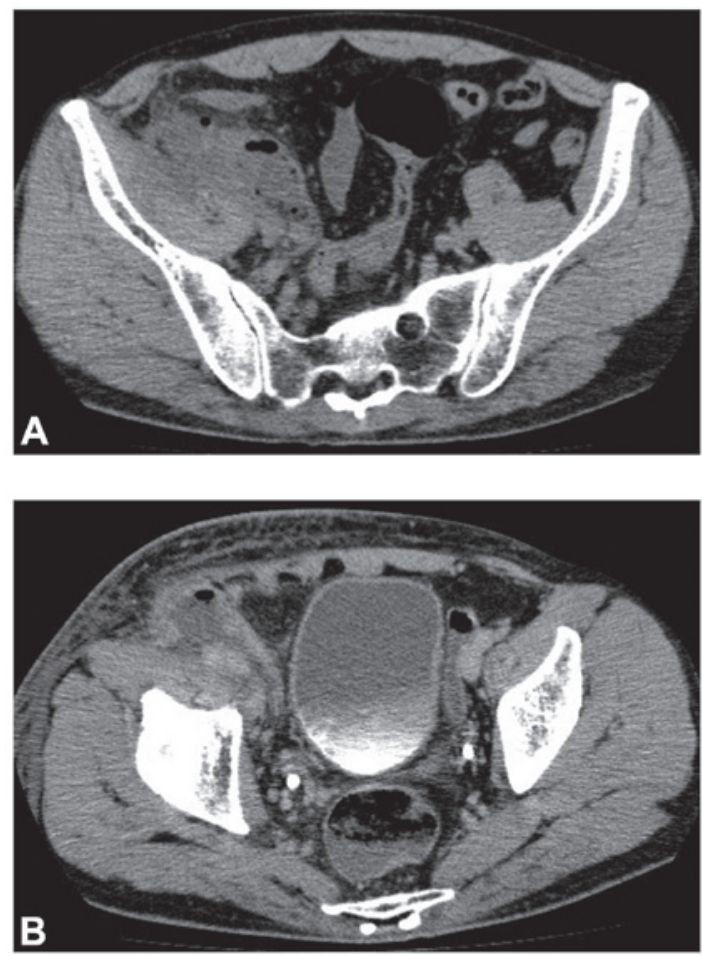

Figure 2. Computed tomography scans of the abdomen revealed fluid and gas collection in the retroperitoneum adjacent to the cecum.

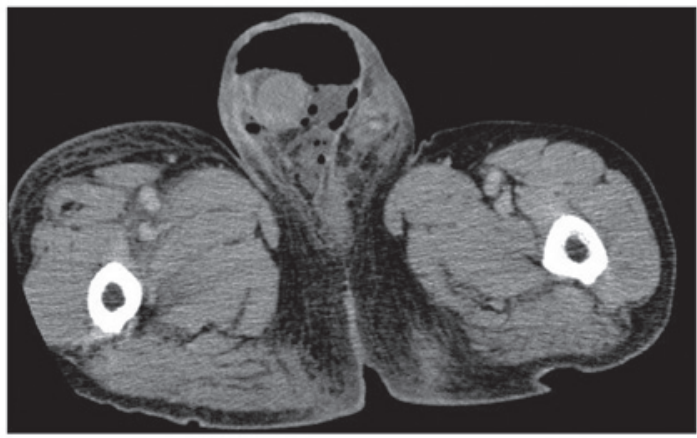

Figure 3. Computed tomography scan of the abdomen revealed a scrotal abscess with gas formation.

Additionally, due to its acute and rapidly progressive course, retroperitoneal abscess is associated with a high morbidity and mortality rate $(1,5,6)$. In the present case, the development of retroperitoneal abscess did not occur as febrile neutropenia. Had this complication developed during myelosuppression, the clinical course may have been more complicated. We initiated administration of intravenous antibiotics immediately after obtaining samples for blood culture. Following an improvement in CRP levels and leukocytosis, a drainage tube was inserted. Recent advances in antimicrobial agents, such as carbapenems and new quinolones, have achieved higher disease control rates, even in severe infections $(9,10)$. The appropriate selection of antibiotics and surgical drainage are considered to be mandatory for patients with retroperitoneal abscesses, whereas surgical debridement is indicated when the abscess is accompanied by symptoms such as intestinal obstruction or bleeding. Our reported case highlights the importance of early diagnosis 
and appropriate treatment in order to avoid subsequent serious complications, such as septicemia and septic shock. Accurate and timely clinical judgment and a careful consideration of the balance between the risks and benefits are required prior to medical and surgical intervention.

Retroperitoneal abscess is a rare but potentially fatal complication in patients treated with chemotherapy for lung cancer, particularly those who are under, or shortly after, chemotherapy-induced myelosuppression. Unexpected infectious complications, such as retroperitoneal abscess, may also develop in patients with lung cancer and physicians should be alert on such a rare complication.

\section{References}

1. Rotstein OD, Pruett TL and Simmons RL: Thigh abscess An uncommon presentation of intraabdominal sepsis. Am J Surg 151: 414-418, 1986.
2. Hall JF, Roberts PL, Ricciardi R, et al: Long-term follow-up after an initial episode of diverticulitis: what are the predictors of recurrence? Dis Colon Rectum 54: 283-288, 2011.

3. Banner MP: Genitourinary complications of inflammatory bowel disease. Radiol Clin North Am 25: 199-209, 1987.

4. Cybulsky IJ and Tam P: Intra-abdominal abscesses in Crohn's disease. Am Surg 56: 678-682, 1990.

5. Panwalker AP: Unusual infections associated with colorectal cancer. Rev Infect Dis 10: 347-364, 1988.

6. Takakura Y,Ikeda S, Yoshimitsu M, et al: Retroperitoneal abscess complicated with necrotizing fasciitis of the thigh in a patient with sigmoid colon cancer. World J Surg Oncol 7: 74, 2009.

7. Maguire J and Kaye SB: Psoas abscess: unusual complication of effective chemotherapy for teratoma. Br Med J (Clin Res Ed) 290: 1870, 1985 .

8. Satoh H, Yano H, Naitoh T, et al: A case of retrocecal appendicitis occurrence during effective chemotherapy in lung cancer. Jpn J Cancer Clin 35: 389-392, 1989 (In Japanese).

9. Rodriguez CL, Ferran NA, Lyons K and Evans RO: Bilateral glenohumeral septic arthritis secondary to retroperitoneal abscess. Bull NYU Hosp Jt Dis 67: 378-380, 2009.

10. Brook I: Urinary tract and genito-urinary suppurative infections due to anaerobic bacteria. Int J Urol 11: 133-141, 2004. 